\title{
Wie bewerbe ich mich richtig?
}

\author{
Dr. Sabine Lang \\ Referatsleiterin in der Abteilung Personal, Stiftung Preußischer Kul- \\ turbesitz, Berlin
}

Für eine erfolgreiche Bewerbung gibt es leider keine Zauberformel und es gelten auch keine definierten Standards. Das mag auf den ersten Blick etwas entmutigend wirken, aber das sollte es nicht. Denn so besteht die Chance, durch eine interessant und ansprechend gestaltete, individuelle Bewerbung das Interesse an der eigenen Person hervorzurufen und beim Adressaten den Wunsch zu wecken, die Bewerberin oder den Bewerber näher kennen zu lernen. Damit dies gut gelingen kann, gibt es immerhin ein paar Regeln, die für eine erfolgreiche Bewerbung hilfreich sein können.

Eine erfolgreiche Bewerbung beginnt mit dem genauen Lesen des Stellenangebots und der Frage „Was wird gesucht, was habe ich zu bieten? “. Hier sollte man sich mit Selbstbewusstsein seiner eigenen Fähigkeiten bewusst werden und diese dann ehrlich, also ohne zu über- oder untertreiben, in seiner Bewerbung darstellen. Wie gelingt das nun am besten?

\section{Bewerbung}

Eine schriftliche Bewerbung sollte folgende Unterlagen enthalten:

- Anschreiben

- Deckblatt (fakultativ)

- Lebenslauf

- Zeugnisse

- und unter Umständen weiteren Unterlagen entsprechend den Anforderungen des Stellenangebots (z. B. Arbeitsproben).

Beim Erstellen des Anschreibens sollten Sie das Ziel klar vor Augen haben, die Aufmerksamkeit des Stellenanbieters zu wecken. Dabei sollte einem bewusst sein, dass bei Verfahren mit hundert und mehr Bewerbungen der Adressat die schwierige Aufgabe zu lösen hat, anhand schriftlicher Unterlagen die am besten geeigneten Bewerber(innen) zu finden. Deswegen gilt, das Anschreiben kurz und präzise zu fassen und im Regelfall nicht mehr als eine Seite zu schreiben. Bereits hier können Sie Ihre Fähigkeit zum prägnanten Formulieren und dem Darstellen des Wesentlichen unter Beweis stellen.

Für das Anschreiben gibt es, wie generell für die Bewerbung, keine Vorgaben, aber am besten wird das Interesse des Lesers oder der Leserin geweckt, indem Sie bereits im ersten Satz kurz darstellen, warum Sie für die Stelle besonders qualifiziert sind. Im Weiteren wird dann belegt, woher die im Einleitungssatz behaupteten Qualifikationen stammen, z.B. dass die besonderen Sprachkenntnisse durch einen Auslandsaufenthalt erworben wurden, die Kenntnisse im Medizinrecht in einem speziellen Seminar oder einer Referendariatsstation. Im Wesentlichen können Sie sich an den Anforderungen der Stellenausschreibung entlanghangeln und auf diese idealerweise spiegelbildlich mit der eigenen Qualifikation erwidern. Günstig ist es, die eigenen Fähigkeiten dergestalt mit den Anforderungen zu verknüpfen, dass deutlich wird, wo der Gewinn für den (die) zukünftige(n) Arbeitgeber(in) liegt, wenn er (sie) einen einstellt. Das heißt: Nach der Lektüre des Anschreibens sollte der (die) Leser(in) wissen, warum er (sie) einen zum Vorstellungsgespräch einladen soll.

Den dann folgenden Unterlagen können Sie ein Deckblatt voranstellen, was nicht zwingend erforderlich ist, aber der Bewerbung einen gewissen Rahmen gibt. Entscheiden Sie sich für ein Deckblatt, ist die Gestaltung natürlich frei. Es empfiehlt sich die Stelle, für die Sie sich bewerben noch einmal zu nennen sowie die wesentlichen persönlichen Daten wie Name, Anschrift, Telefonnummern und E-Mail-Adresse anzugeben. Einladend wirkt auch ein Bild auf dem Deckblatt.

Ein wesentlicher Bestandteil der Bewerbung ist der Lebenslauf. Hier bietet sich die Möglichkeit, seine individuellen und besonderen Fähigkeiten und Erfahrungen darzustellen. Es lohnt sich hier einmal viel Mühe für die optimale Variante zu investieren, da der Lebenslauf Sie immer begleiten wird. Auch sollten Sie die Bedeutung des Lebenslaufes nicht unterschätzen, da er dazu dient, auf einen Blick den Werdegang der Bewerberin oder des Bewerbers zu erfassen.

Es gibt auch für das Erstellen des Lebenslaufs keine vorgegebenen Standards. Bewährt hat sich meines Erachtens die Variante der „verkehrten Darstellung“, das heißt, man beginnt mit den letzten Lebensereignissen. Allem vorangestellt werden natürlich die persönlichen Angaben, Name, Geburtsdatum und -ort, Adresse, Staatsangehörigkeit, fakultativ Familienstand und Kinder.

Für die Darstellung des beruflichen und schulischen Werdegangs bietet sich eine Unterteilung in berufliche Erfahrungen (soweit vorhanden), Referendariat, Studium, Schulabschluss, besondere Qualifikationen an. Angaben zu Hobbys und Freizeit sollten meines Erachtens nur bei besonderen Hobbys erfolgen oder wenn die Freizeitgestaltung einen Bezug zur angestrebten Tätigkeit hat. So ist es bei einer Bewerbung als Verwaltungsleiter(in) einer Opernstiftung durchaus von Interesse, im Opernchor zu singen.

Sinnvoll ist es, die einzelnen Punkte des Lebenslaufes kurz zu beschreiben. So sollten Sie besonders interessante Referendariatsstationen erwähnen oder bei beruflichen Tätigkeiten die wesentlichen Aufgaben benennen. Bei der Unmenge an Berufsbezeichnungen ist es ansonsten aus Adressatensicht oftmals schwer zu erkennen, was sich dahinter tatsächlich verbirgt.

Dem Lebenslauf folgen Zeugnisse und sonstige Unterlagen. Unerlässlich sind Examens- und andere Abschlusszeugnisse wie beispielsweise Masterabschlüsse oder Promotionsurkunden. Soweit vorhanden, müssen auch Beurteilungen und Zeugnisse bisheriger Arbeitsgeber(innen) beigefügt werden. Da man zu Beginn des Berufslebens über derartige aussagekräftige Unterlagen nicht verfügt, können auch Praktikumsbeurteilungen oder andere Bescheinigungen hilfreich sein. Um die Auswahl der Unterla- 
gen möglichst ideal zu treffen, sollten Sie sich Sinn und Zweck dieser Unterlagen verdeutlichen. Diese besteht darin, im Anschreiben und Lebenslauf behauptete Qualifikationen durch Dritte bestätigt zu finden. Sie sollten eine kluge und bestimmte Auswahl treffen, da Sie somit auch nochmals besondere Fähigkeiten unterstreichen können. Auf alle Fälle sollten Sie Beliebigkeit vermeiden.

Schulzeugnisse sollten Sie nur beifügen, wenn diese ausdrücklich erwünscht sind.

Weitere Unterlagen, wie Arbeitsproben, werden bei Juristen eher selten verlangt. Bewirbt man sich jedoch in weniger klassischen Juristenberufen, wie beispielsweise als Journalist(in), können diese durchaus von Bedeutung sein.

Ein Tipp zum Schluss: Auf keinen Fall Musterschreiben aus dem Internet verwenden. So zahlreich sie dort auch zu finden sind, sie können nur Anregungen und Ideen bieten, mehr aber nicht.

\section{Vorstellungsgespräch}

Haben Sie eine Einladung zum Vorstellungsgespräch erhalten, haben Sie die erste Hürde des Bewerbungsverfahrens erfolgreich genommen. Das kann Sie zu Recht stolz machen und Ihnen genügend Selbstvertrauen für die nächste Runde geben. Eine gute Vorbereitung auf ein Vorstellungsgespräch ist immer möglich und verleiht auch eine gewisse Sicherheit.

Zuerst sollten Sie alle verfügbaren Informationen über den (die) mögliche(n) künftige(n) Arbeitgeber(in) einholen. Hierfür ist das Internet eine oftmals hilfreiche und wertvolle Informationsquelle. Aber auch über künftige zu erwartende Aufgaben sollten Sie sich informieren. Je mehr Informationen, desto besser.

Vorstellungsgespräche beginnen in der Regel mit der Bitte, sich kurz vorzustellen und die Gründe für die Bewerbung zu nennen. Es mag Varianten geben, meist werden diese Fragen aber gestellt, so dass es sich lohnt, die Antworten zu diesen Fragen schon einmal zu überdenken. Den eigenen Werdegang sollten Sie dabei kurz und prägnant darstellen mit besonderem Schwerpunkt auf Stationen im Lebenslauf, die für die Stelle relevant sind. Für die Darstellung der Motivation für die Bewerbung können Sie gut die über Aufgaben und Arbeitgeber(in) herausgefundenen Informationen nutzen. Der weitere Verlauf des Ge- sprächs hängt dann im Wesentlichen von dem (der) Gesprächsführer(in) $\mathrm{ab}$, ist aber mit einer guten Vorbereitung zu meistern.

Natürlich können Sie auch in einem Bewerbungsgespräch Fragen stellen. Jedoch sollten Sie tatsächlich nur nach Sachverhalten erfragen, die von wirklichem Interesse sind. Wenn Sie keine Fragen haben, womöglich weil auch seitens des (der) Arbeitgebers (Arbeitgeberin) alle Informationen gegeben wurden, sollten Sie sich nicht Fragen um der Fragen willen ausdenken.

Auch hier ein Tipp zum Schluss: Keine Angst vor der eigenen Aufregung haben. Ein bisschen Aufregung schadet nicht, sondern macht eher sympathisch.

\section{Was tun, wenn es mit der Bewerbung nicht geklappt hat?}

Die Antwort auf diese Frage klingt simpel, sollte aber unbedingt beherzigt werden: Nicht verzagen!

Zunächst sollten Sie sich selbst noch einmal fragen: War es tatsächlich die richtige Stelle für mich? Zu Beginn seiner Berufskarriere neigt man dazu, sich eher breit als gezielt zu bewerben. Waren beispielsweise bei der Stelle mindestens zwei Jahre Berufserfahrung verlangt, sollte das einen nicht unbedingt abhalten sich zu bewerben, aber eine Absage ist dann eben wahrscheinlicher als eine Zusage.

Kommen Sie zu dem Schluss, Sie haben alle Anforderungen in idealer Weise erfüllt und Sie waren dennoch nicht erfolgreich, so kann ich aus Personalabteilungssicht sagen, dass man in manchen Verfahren gerne drei oder vier Bewerber(innen) einstellen würde. Aber wenn nur eine Stelle zur Verfügung steht, muss eine Entscheidung getroffen werden. Solche Situationen sind für alle Seiten bitter, aber kein Grund, an der eigenen Qualifikation zu zweifeln.

Hilfreich kann es sein, im Fall einer Absage nachzufragen, woran es gelegen hat. Wie auskunftsbereit die jeweiligen Arbeitgeber(innen) sind, hängt stark von der Unternehmenskultur ab. Hatten Sie ein gutes Gefühl im Vorstellungsgespräch, sollten Sie sich nicht scheuen anzurufen.

Sollte auch nach einer Vielzahl von Bewerbungen noch keine erfolgreich gewesen sein, dann sollte man dennoch nicht aufgeben. Jede Bewerbung, jedes Vorstellungsgespräch ist eine gute Übung, die sicherer macht und irgendwann auch ganz bestimmt zum Erfolg führt.

\section{Geburtstage}

(April bis Juni 2010)

\section{Jahre}

Gisela Forst
Vorsitzende Richterin am
BPatG i.R.
München
Dr. Ingeborg Franke
Vizepräsidentin des BVG a.D.
Berlin

$\begin{array}{ll}\text { Ingrid Horstkotte } & \mathbf{8 5} \text { Jahre } \\ \text { Direktorin des AG i.R. } & \\ \text { Hamburg } & \text { Hella Klose-Frantzen } \\ & \text { Rechtsanwältin i.R. } \\ \text { Karin Kausch-Blecken von } & \text { Kleinmachnow } \\ \text { Schmeling } & \\ \text { Richterin am OLG i.R. } & \\ \text { Cully (Schweiz) } & \\ \text { Marie-Luise Schmidt } \\ \text { Staatsanwältin a.D. } \\ \text { Oldenburg }\end{array}$

